the triad of ptosis, miosis and anhidrosis. In the current literature, the development of HS after interscalene brachial plexus block is $4-37.5 \%$, depending on the block technique, distribution, volume and dilution of the local anesthetic, in continuous infusion or after a single injection. Data showed that the use of ultrasound and neurostimulator to perform the block can reduce the rate of $\mathrm{HS}$.

\section{RESULTS OF COMBINED POPLITEAL AND SAPHENOUS NERVE BLOCK APPLICATIONS}

H Emirkadi* i Çiçek. Gölcük Necati Çelik Goverment Hospital, Kocaeli, Turkey

10.1136/rapm-2021-ESRA.190

Background and Aims Peripheral nerve block techniques for extremity surgeries have gained increasing popularity and have been used often in recent years. With the help of this method, much more comfort can be provided without the need of general anesthesia.Successful surgery and postoperative analgesia can be obtained by detecting accurate anatomic localization, dermatome and myotomes.

Methods We studied 35 patients (27 male, 8 female)retrospectively,whom received combined sciatic popliteal and saphenous nerve block using peripheral nerve stimulator for lower extremity surgeries between 2018-2020. Diabetic patients were excluded from the study due to diabetic neuropathy. Patients were sedated with $1 \mathrm{mg}$ midazolam before the blockage.Then,the sciatic nerve was localized by peripheral nerve stimulator and popliteal sciatic block was performed by 20 $\mathrm{mL}$ of $0.25 \%$ bupivacaine and, $20 \mathrm{~mL}$ of $1 \%$ lidocaine.Saphenous vein blockage was performed by $5 \mathrm{ml}$ of $2 \%$ lidocaine and $5 \mathrm{ml}$ of $0.5 \%$ bupivacaine.

Results The mean blockage performing time was determined as 30 minutes. A tourniquet was applied above $5 \mathrm{~cm}$ at the ankle joint for all operations. Average tourniquet (bound) time was measured as 40 minutes. None of the patients needed additional anesthesia procedures.Significant intraoperative complications associated with the application were not observed in the postoperative period.Degree of the patients' post-operative pain was assessed using Visual Analogue Scale.Patients did not require narcotic analgesics in the early and late postoperative

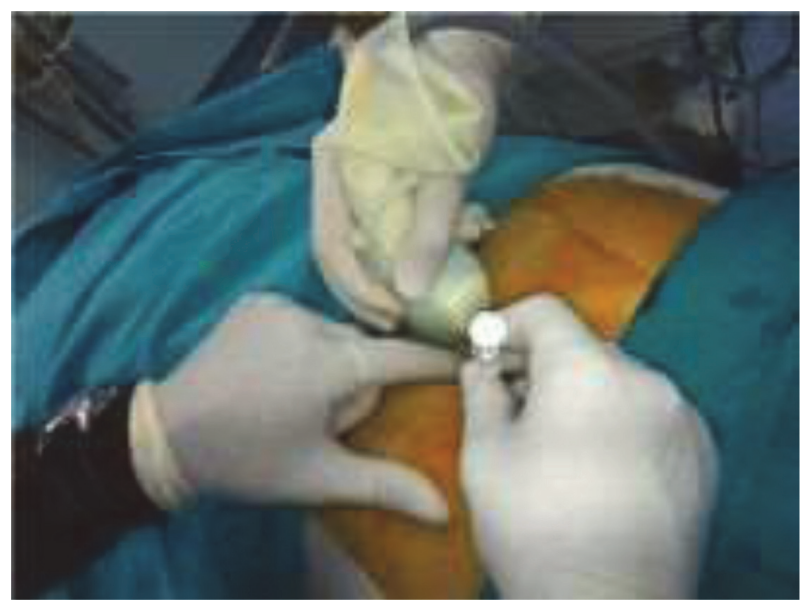

Abstract 190 Figure 1
period.In all patients,oral intake was started after the completion of surgery

Conclusions In addition to all these advantages, we believe that combined popliteal sciatic block should be preferred in foot surgery for selected patients to avoid complications of general or spinal anesthesia.

\section{A NEW MODIFIED TECHNIQUE OF COMBINED INTRAMUSCULAR QUADRATUM LUMBORUM BLOCK AND IT'S EFFECTIVENESS IN PEDIATRIC PATIENTS}

${ }^{1}$ D Dmytriiev*, ${ }^{2} E$ Glazov. 'Vinnitsa National Medical University, Vinnitsa, Ukraine; ${ }^{2}$ Odessa Regional Children Hospital, Odessa, Ukraine

\subsection{6/rapm-2021-ESRA. 191}

Background and Aims Regional anesthesia has a rapidly growing use in children over the recent two decades. An aim of our work is to suggest a new technique for quadratum lumborum block (QLB) and prove its efficacy compared to intravenous anesthesia in children.

Methods 44 children (12.6 \pm 1.4 years) were included into the study. First group - 20 patients - received anesthesia by new QLB technique (figure 1) under ultrasound guidance with $0.5 \%$ bupivacaine hydrochloride.

20 children received a continuous fentanyl intravenous infusion. Efficacy of postoperative analgesia was assessed through the complex of different vital signs; pain intensity was assessed by the Observation Scale for Infants and Small Children (VAS). Ethics Committee approved the conduct of the study. Statistical analysis was performed by a variation statistics method using 'Statistica 5.5 ' soft and visualized through soft based on Python „Plotus XYZ'.

Results The average pain score according to VAS was significantly lower in patients of the first group in early postoperative period at all stages of the study $(p<0.05)$. The average heart rate was lower than $120 \mathrm{bpm}$ in group 1 for the entire time of QLB. Parameters of central hemodynamics, arterial blood flow in superior mesenterial artery and glucose levels were also normal.

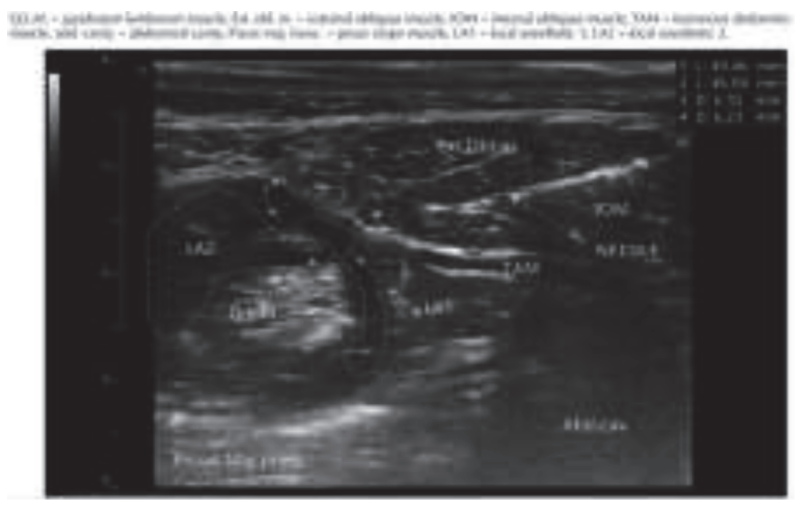

Abstract 191 Figure 1

Conclusions Obtained data shows, that proposed new QLB technique is more effective, when compared to standard intravenous anesthesia. 